\title{
Modelling and Control of a Robotic Arm Using Artificial Neural Network
}

\author{
Satyajit Bhowmick ${ }^{1}$, Rajesh Bag ${ }^{1}$, SK Masud Hossain ${ }^{1}$, Subhajit Ghosh ${ }^{1}$, \\ Shanta Mazumder ${ }^{1}$, Sauvik Das Gupta ${ }^{2}$ \\ ${ }^{I}$ Department of Electronics and Communication Engineering, WBUT, Kolkata, India \\ ${ }^{2}$ School of Electrical and Computer Engineering, Oklahoma State University, OK, USA
}

\begin{abstract}
Often it can be seen that men with a lost arm face severe difficulties doing daily chores. Artificial Intelligence could be effectively used to provide some respite to those people. Neural networks and their applications have been an active research topic since recent past in the rehabilitation robotics/machine learning community, as it can be used to predict posture/gesture which is guided by signals from the human brain. In this paper, a method is proposed to estimate force from Surface Electromyography (s-EMG) signals generated by specific hand movements and then design and control a Robotic arm using Artificial Neural Network (ANN) to replicate human arm. Here the force prediction is a Regression process. A hand model has been successfully moved using servo motor that has been programmed based on the results obtained from sample data. The results shown in this paper illustrate how the Robotic arm performs.
\end{abstract}

Index Terms: Surface EMG, Artificial Neural Network, Robotic arm, Regression.

\section{Introduction}

This project has been designed on data taken as Surface EMG signals from the human arm. Surface Electromyography is a non-invasive technique for measuring muscle electrical activity that occurs during muscle contraction and relaxation cycles. EMG signals contain the information about the muscle force which can be used in human-machine interaction. Force plays an important role in these applications. Rezazadeh et al. [1] proposed a co-adaptive Human-Machine Interface (HMI) that is developed to control virtual forearm prosthesis over a long period of operation. This paper has influenced us to make a robotic arm based on the hand EMG signal. The physical structure of the robotic arm has been modeled such that it can be easily assembled. It has been simplified such that it has 1 degree of freedom (with capability of rotating $180^{\circ}$ ) while retaining some of the important motions of the human arm. A five-finger model has been constructed. The working is based on the simple fact of comparison of performances of EMG signals taken from human arm and the target to be achieved. This model exhibits the full range of motion required to move the arm with some degree of force. A virtual reality model package has been developed in MATLAB to control the model in such a manner that it is able to demonstrate the potential of the work. Mobasser et al. [2] used multilayer perceptron ANN for hand force estimation from surface EMG signals for applications in sports activities. Yang et al. [3] demonstrated the use of ANN, Locally Weighted Projection Regression (LWPR) and SVM to estimate hand grasp force from surface EMG signals for force control of multi-functional prosthetic hands. Haritha et al. [4] used a method to estimate the hand force from Surface Electromyography signals using ANN.In this work, we will estimate the predicted value of force from the surface EMG signals using a feed-forward ANN. The neural network is trained with both EMG and force data. Then the arm model is moved using servo motor which is programmed based on the results obtained from sample data. The next section describes the hardware setup of our system. Section III presents the methodology we proposed for force estimation and operation of the robotic arm. Section IV discusses the experimental evaluation and results. Section V presents the conclusion of the paper.

\section{Hardware Platform}

This Module details about the hardware components used in this work. The hardware platform of our research consists of surface electrode, amplifier, data acquisition board and arm model. Below we will discuss in more details about the individual components.

\section{A. ELECTRODE}

The electrodes are used to collect muscle signals. We are using surface gelled disposable electrodes. They come in various 
(a)

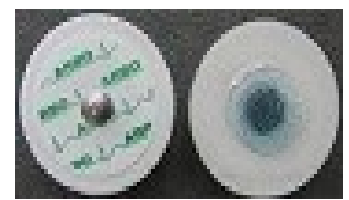

(b)

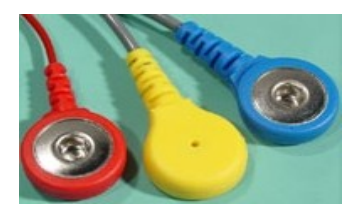

Figure 1. (a) Kendall Arbo skin surface electrode and (b) Snap-on electrode cable

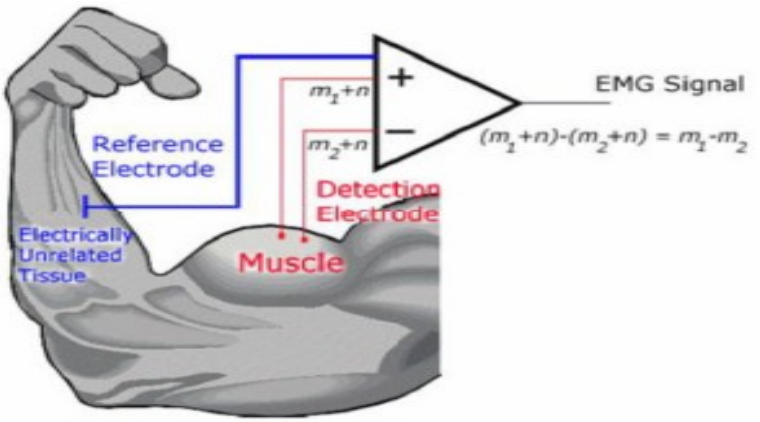

Figure 2. Schematic of the EMG extraction along with differential amplifier configuration

shapes and sizes. They are very light. We use the Kendall Arbo [5] skin surface electrodes in this project as the sensing electrodes. We also have used Snap-On type electrode cable to transmit EMG signals to the amplifier.

\section{B. AMPLIFIER}

EMG is a low amplitude signal and raw EMG data from the muscle cannot be read or used for further processing without amplifying. Hence, an amplifier is connected to the electrodes to get measurable and process-able EMG data. In our project

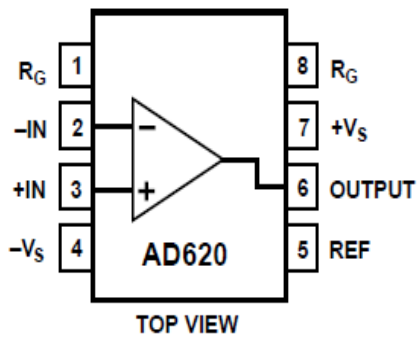

Figure 3. Connection diagram of AD620

we have used Instrumentation amplifier AD620.It is a low cost, high accuracy instrumentation amplifier that requires only one external resistor to set gains of 1 to 1000.The low noise, low input bias current and low power of the AD620 make it well suited for medical Applications such as ECG and non-invasive blood pressure monitors.

\section{DATA ACQUISITION BOARD}

The amplified data is acquired by NI USB-6008 [6] data acquisition device and used in this work for collecting EMG signals. It is an Analog to Digital Converter (ADC) DAQ manufactured by National Instruments. The picture of the device is shown in the section below.

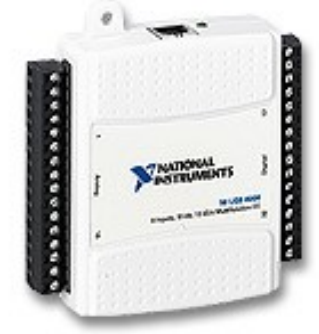

Figure 4. NI USB-6008 
This device is connected to the PC by a USB cable.

We need EMG and Force data to go further. Forces are collected and pre-saved from a Novint Falcon haptic device, along with the s-EMG. The Novint Falcon is a haptic device that has a grip or handle that the user can hold to control it. The grip moves in three dimensions (right-left, forwards-backwards and up-down). However, only the force in the $\mathrm{Z}$ direction is considered to represent the force produced through wrist flexion of the hand. The force is normalized to match EMG for further analysis. When the grip is moved, the Falcon's software tracks the movements and sends current to the motor which in turn creates a force that a user can feel. These EMG and Force data samples are utilized.

\section{ARM MODEL}

The robotic arm consists of a wooden base connected to another wooden part which is made to resemble the human part from the shoulder to the elbow. The servo motor is placed at the elbow which is clamped to a hand made up of light metal sheet, covered with crape bandage and a five finger model of cardboard.

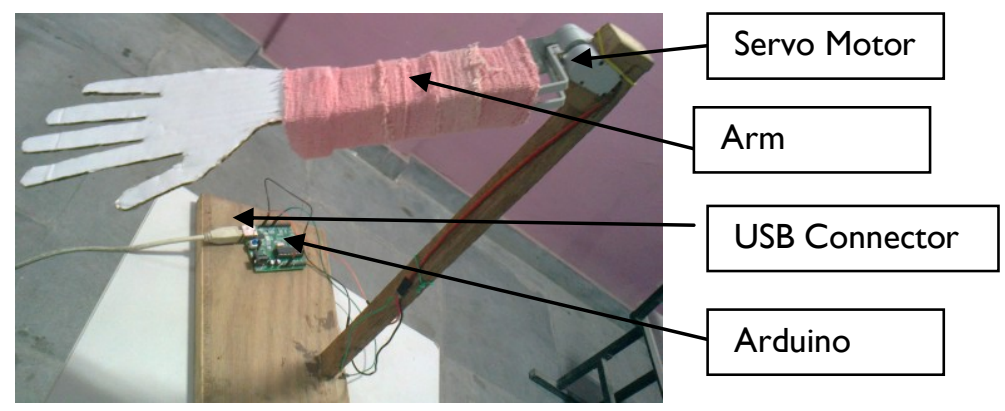

Figure 5. Robotic arm model

A servomotor is a rotary actuator that allows for precise control of angular position. It consists of a motor coupled to a sensor for position feedback, through a reduction gear box. We have used servomotor VS1 [7]. The entire model is made to mimic a human arm. The logic control of the arm is entirely done using an Arduino board placed at the arm's base. Arduino [8] is a single-board microcontroller designed to make the process of

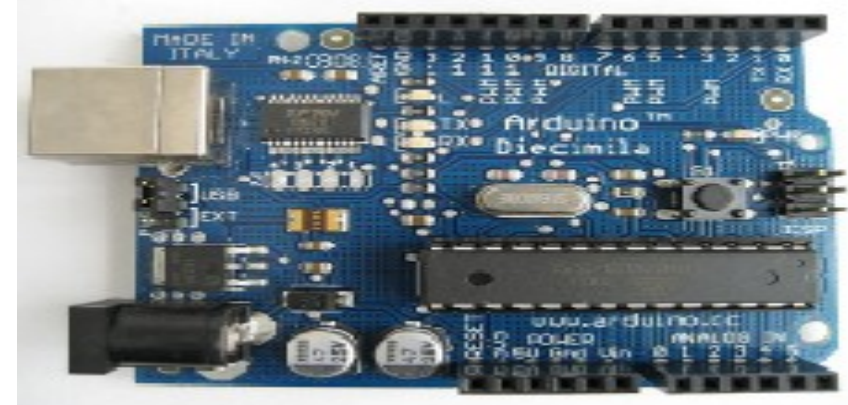

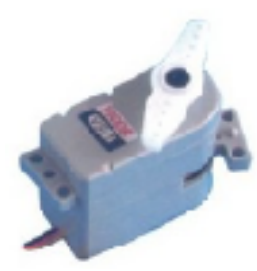

(b) (a)

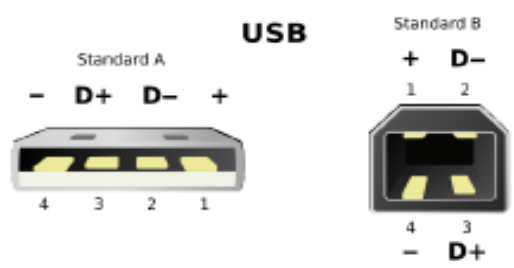

(c)

Figure 6. (a)Arduino decimilia and (b) Servo motor VS1 and (c) The standard USB A plug (left) and B plug (right)

using electronics in multidisciplinary projects more accessible. The arm is made as light as possible because the servomotor can take maximum of 200 grams of weight, so the lighter the arm, the better it is. USB connector is used to connect the arduino to the computer \& Serial Communication protocol is used to establish communication between MATLAB \& arduino microcontroller. The basic USB connection is of two male jacks viz. type A and type B. 


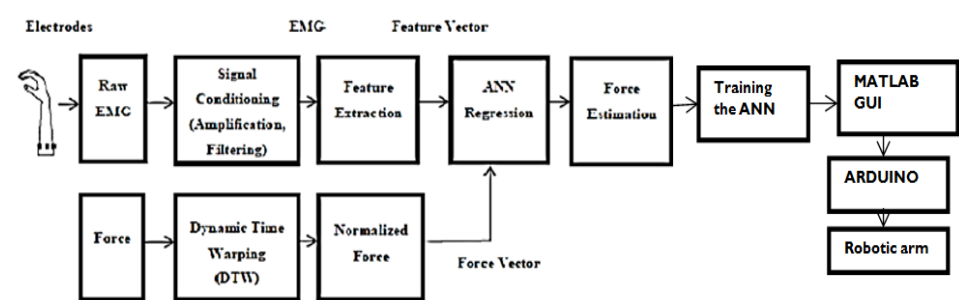

Figure 7. Overall method for Movement of the Robotic Arm

\section{Methodology}

After Data Acquisition, the whole procedure till operating and moving the robotic arm can be broadly classified into three steps, namely pre-processing, training the ANN and compilation. Before describing the methods, it should be mentioned that we are using data samples, previously collected from falcon. The samples are not discrete, they are in matrix form. We took five data samples of EMG vector and corresponding 5 samples of equivalent force vectors. The co-relation is done between sample EMG of matrix (1x3311) and sample Force of matrix (1x3311).

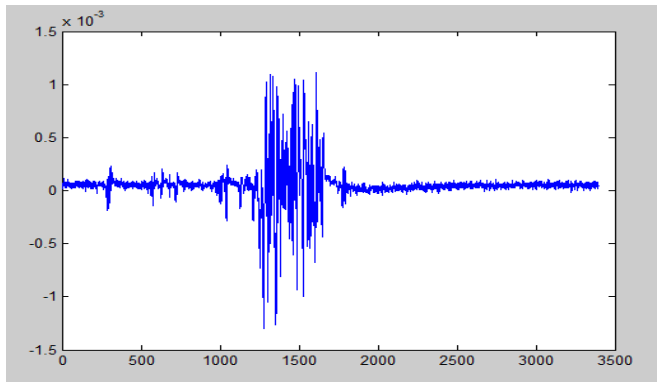

Figure 8. Raw EMG signal

\section{A. PRE-PROCESSING}

\section{i. Feature Extraction}

This step extracts important information from EMG signals. Root Mean Square (RMS) is used as the feature extraction technique in this work \& eliminates the dual-axis nature of the acquired EMG data. The mathematical equation for RMS is given as follows:

$$
R M S=\operatorname{sqrt}\left(\left(\frac{1}{N}\right) \sum_{i=1}^{N} x_{i}^{2}\right) \quad \cdots \cdots \cdot 1[\square
$$

Wherle,

$\mathrm{N}=$ Total number of values

$\mathrm{x}=$ Individual data points

A MATLAB code is written in order to get the dynamic RMS values of the EMG signals through an overlapping continuous

window update method for the whole data length of the EMG. The value of the window (N) is fixed to 90 samples in this case

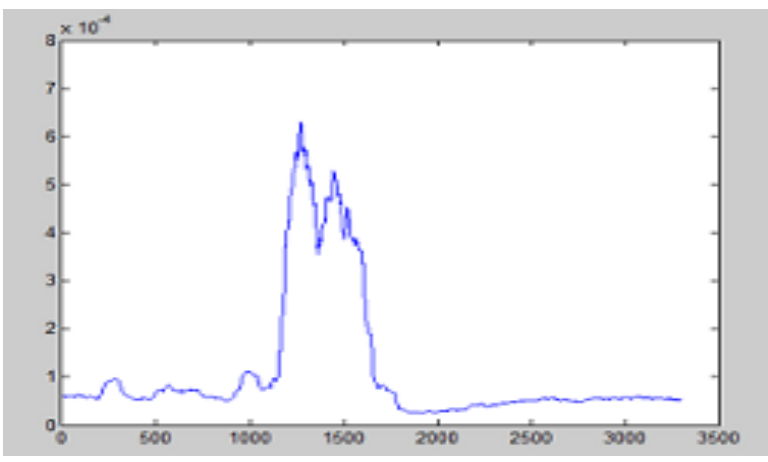

Figure 9. RMS plot of EMG data 


\section{ii. Data filtering}

Raw EMG data needs to be filtered in order to reduce the noise or improve the Signal-to-Noise Ratio (SNR). Smoothing filter is applied on the signal to remove the high frequency fluctuations. Here we implement a Pseudo-Gaussian smoothing filter in MATLAB to achieve the above purpose. The noise is considerably reduced and the SNR is improved which in turn facilitates better feature extraction. Seeing the figure we can understand the importance of this step.

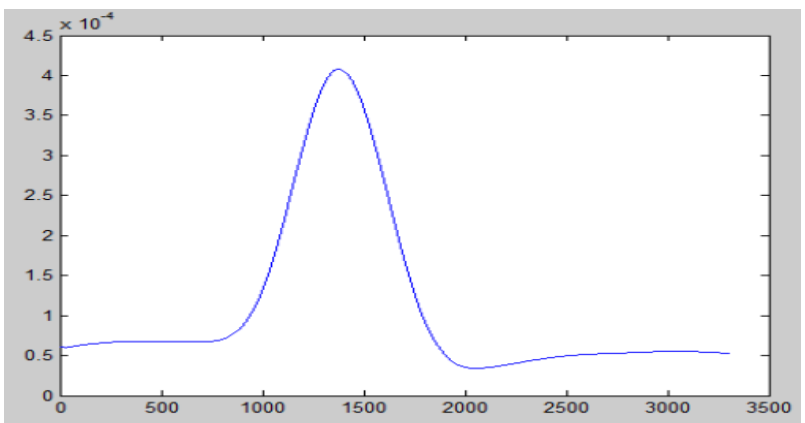

Figure 10. RMS plot of EMG data after filtering

\section{iii. Transformation}

Even though, filtered EMG and force values were recorded at the same time and for the same amount of time, Force transformation or normalization was done by a MATLAB program using the mapstd function in order to overcome the problem of mismatch in the scale of samples of force values (in Newtons) and EMG values (in Millivolts). It process matrices by mapping each row's mean to zero and deviations to one. It is an approach for scaling network inputs and targets so that they will have zero mean and unity standard deviation. The input and the target vector to the neural network are the feature vector of EMG values and normalized force vector values. The regression results are improved.

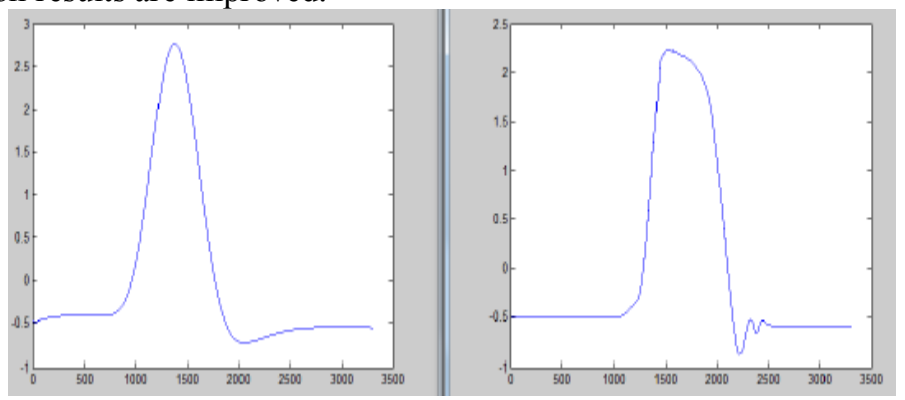

Figure 11. Normalized EMG data on left and normalized force on right

\section{iv. Dynamic Time Warping}

Dynamic Time Warping (DTW) is a well-known technique to find an optimal alignment between two given (time-dependent) sequences. It gives nonlinear time normalization between the two signals. DTW can be applied to two waveforms which have approximately the same overall amplitude profiles, but are not close to each other in the time axes. In spite of the actions being the same, it is impossible to repeat the same action with same intensity \& for the same time-span. Hence, in some actions, though the peak values of forces are same, the time axes do not match. DTW was used to find an optimal alignment with time series and to improve regression results. Reference templates for DTW are created by averaging all the signals. DTW is used to eliminate the distortion in these signals.

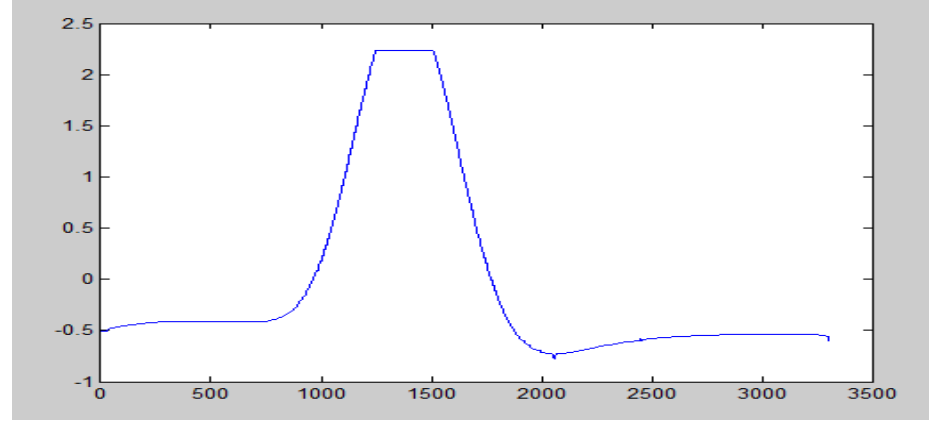

Figure 12. Force data after DTW 


\section{B. TRAINING THE ANN}

In this work, a two layer feed forward artificial neural network with sigmoid hidden neurons and linear output neurons are used for force regression from a single channel of EMG signal. The network is trained with data samples for flexions. The input to the feed forward neural network is the EMG feature vector and the force is the target vector (We have taken data samples of force). In order to improve the performance of the network and to prevent high bias and variance, the data is separated into training set, cross-validation set and testing set. The network is trained with four different sets of inputs (different sets of data obtained from a single channel of EMG) and targets (corresponding force values).The Mean Squared Error (mse) is used as the performance measure. The average training error is found to be $3.05355 \mathrm{e}-05$. Average overall regression value from training, validation and test set is found to be 0.9999 (maximum value of $\mathrm{R}$ is 1).

\section{COMPILATION in MATLAB GUI}

In this automated callback function, we introduce our required code.

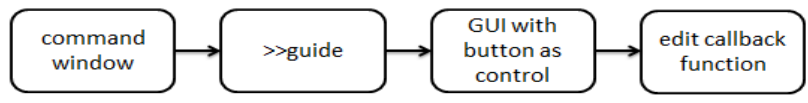

Figure 13. Flowchart of basic steps to make a Matlab GUI

We have constructed a MATLAB GUI in order to simplify the workflow through graphical control \& avoid using huge commands repetitively.

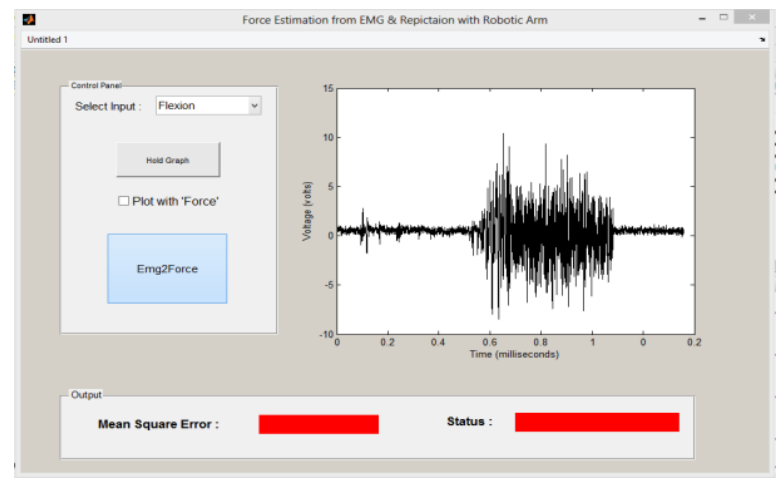

Figure 14. The final GUI looks like this

\section{i. Interfacing and MSE calculation}

After the serial port is made available for duplex communication between MATLAB \& Arduino, we check the mse. If the mse is lower than a certain threshold value to match the original signal, then the movement of motor will be clockwise or counter clockwise as programmed. Otherwise it will not rotate. By observation, we found this threshold mse value to be 0.05 . For our test case, we considered the Flexion movement as our test case. Movement can be in both ways with greater prospects.

\section{ii. Compilation Workflow}

- The mse obtained from ANN is checked.

- If mse is less than 0.05 , MATLAB communicates with Arduino with a baud rate of $9600 \mathrm{bits} / \mathrm{sec} \&$ sends a go-ahead flag signal.

- If Arduino receives the go-ahead flag, it commands the servo to rotate at an angle $60^{\circ}$ and again return to its original position.

- Thus flexion is replicated.

- If mse is greater than 0.05 , it implies, the obtained signal is varied too much from the original signal to be considered as flexion. Thus no action is taken.

\section{Experimental Evaluation}

First of all, the muscle site, the flexor carpi ulnaris muscle is identified to be involved in the flexion action. The selected hand area is cleaned with NuPrep abrasive skin cream [9] to remove dry and dead cells in order to increase signal conduction and skin electrode impedance. Ten20 conductive enhancement paste [10] is applied to the grey portion of the EMG electrode to enhance conduction. The electrode is connected to the amplifier through electrode cable. This amplifier through NI USB-6008 is connected to the computer. Using this 
arrangement we have collected the EMG data samples for different hand movement using DAQ software and MATLAB. The second part of the experiment is to operate the robotic arm using the flexion EMG samples. In this part, we connect the arm using USB connector to the computer. We have randomly chosen three samples, one for each of flexion (bending of arm from elbow), grasp (grasp of palm) and wave (wrist waving) movements. We have got the mse for those samples, using the previously mentioned methods, as follows:

Table 1: Mean square error between actual and predicted force

\begin{tabular}{|l|c|}
\hline Action & MSE \\
\hline Flexion & $3.50355 \mathrm{e}-5$ \\
\hline Grasp & 0.095 \\
\hline Wave & 0.074 \\
\hline
\end{tabular}
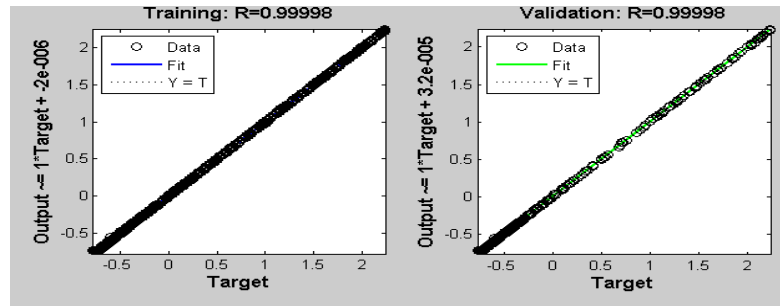

Test: R=0.99997

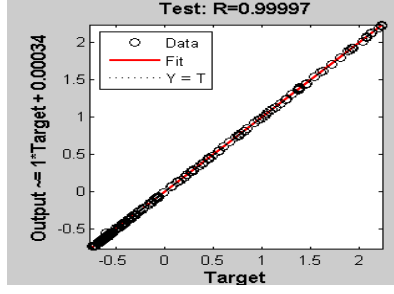

All: $\mathbf{R}=0.9999$
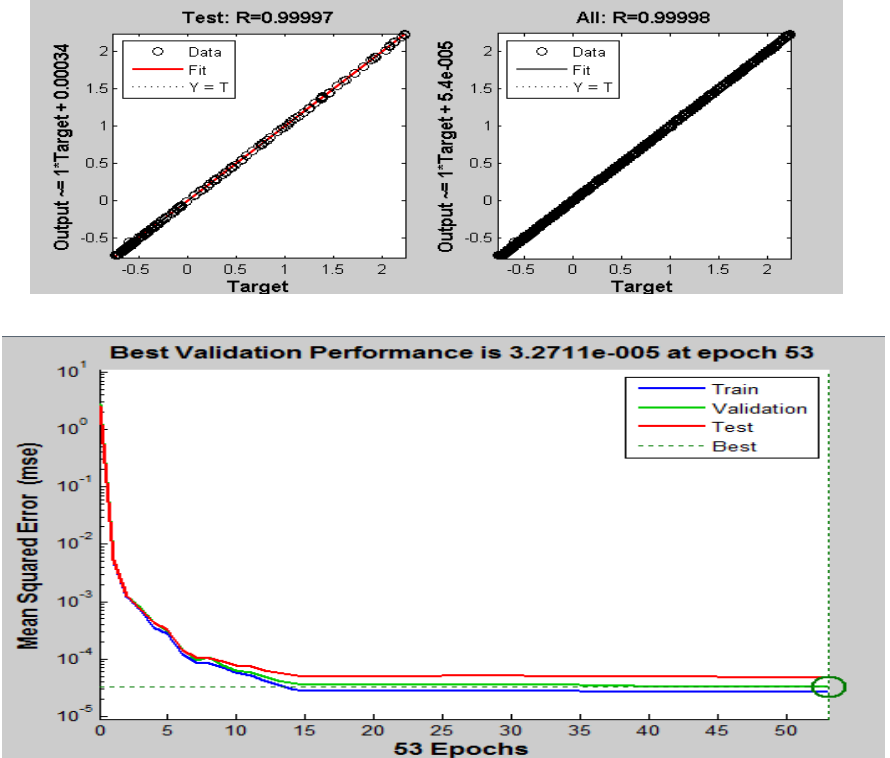

(b)

Figure15. (a) Regression curve for flexion and (b) validation performance curve for flexion (Training curve, validation curve, test curve, best fitting curve)

As force is correlated with flexion, grasp and wave show no correlation. So, mse for grasp and wave is higher. We have set the value of the mse, 0.05 , as the condition for movement of the arm. If mse is less than 0.05 , then there will be a movement of the arm (from stable state to upwards or downwards direction and then back to the stable state). When we run the Matlab GUI then we found that the robotic arm moved UP and DOWN only for the input EMG of flexion as this is the best fitting curve, matches closely with the force and the mse is within the range. For the rest of the signals the arm does not move as mse does not match with that of the condition.

\section{Discussions And Conclusions}

This paper presents a method to estimate the muscle force from the surface EMG signals using ANN and replicate it through a robotic arm. It is a very basic model. Here we have collected data from only one muscle as involving different muscle would make it more complicated. The network trained for one subject may not be applied for other subjects since the muscle force exerted may not be the same for all the subjects. Hence, the network has to be re-trained when the subject changes. Moving on to the part of replication through robotic arm, current generation of robotic arms generally contains six degrees of freedom. But for the sake of simplicity, we have considered only one. Moreover, only the arm joint of the hand has been replicated, while joints of fingers 
have been ignored. A force feedback system would also increase sensitivity. Though there are some shortcomings, the model could effectively be used in future with some modifications and advancement for purposes like prosthetic arm, force multiplication using EEG signals etc.

\section{Acknowledgement}

This project is supported by WBUT and ESL, Salt Lake, Kolkata.

\section{References}

[1] I.M. Rezazadeh, M. Firoozabadi, H. Hu and M. Reza Hashemi Golpayegani, "Co-Adaptive and Affective Human-Machine Interface for Improving Training Performances of Virtual Myoelectric Forearm Prosthesis", IEEE Transactions on Affective Computing, Vol. 3, No. 3, 2012, pages 285-297.

[2] F. Mobasser and K. Hashtrudi-Zaad, "Rowing Stroke Force Estimation with EMG Signals using Artificial Neural Networks", in Proc. 2005IEEE Conference on Control Applications, Toronto, Canada, August 28-31, 2005, pp. 825-830.

[3] D. Yang, J. Zhao, Y. Gu, L. Jiang and H. Liu, “Estimation of Hand Grasp Force based on Forearm Surface EMG”, in Proc. 2009 IEEE International Conference on Mechatronics and Automation, Changchun, China, August 9-12, pp. 1795-1799.

[4] Haritha Srinivasan., Sauvik Gupta, Weihua Sheng and Heping Chen, "Estimation of Hand Force from Surface Electromyography Signals using Artificial Neural Network", The $10^{\text {th }}$., World Congress on Intelligent Control and Automation (WCICA), 2012, Beijing, China, 6-8 July 2012, pages 584-589.

[5] "Kendall ARBO disposable EMG/EKG Electrodes", [Online], Available:http://bio-medical.com/products/kendall-tycoarbodisposable-electrodes-50pkg.html

[6] "NI USB-6008 DAQ", [online], Available: http://sine.ni.com/nips/cds/view/p/lang/en/nid/201986

[7] Servomotor http://en.wikipedia.org/wiki/ServoMotor

[8] Arduino http://www.arduino.cc/

[9] "NuPrep skin preparation gel" [Online].Available: http://biomedical.com/products/nuprep-eegecg-skin-prep-gel-3-pack-of4oztubes.html

[10] "Ten20 Conductive Paste" [Online]. Available: http://www.doweaver.com/ten20.html 